\title{
Multi-component dark matter from a hidden gauged SU(3)
}

\section{Stephen Godfrey* and Alexandre Poulin}

Ottawa-Carleton Institute for Physics, Carleton University, 1125 Colonel By Drive, Ottawa, Ontario K1S 5B6, Canada

E-mail: godfrey@physics.carleton.ca, alexandre.poulin@carleton.ca

\begin{abstract}
We studied Dark Matter (DM) phenomenology with multiple DM species consisting of both scalar and vector DM particles in the Hidden Gauged SU(3) model of Arcadi et al. Because of the large parameter space in the Hidden Gauged SU(3) model we restrict ourselves to three representative benchmark points, each with multiple DM species. The relic densities for the benchmark points were found using a program developed to solve the coupled Boltzmann equations for an arbitrary number of interacting DM species with two particles in the final state. For each case, we varied the mass of the DM particles and then found the value of the dark SU(3) gauge coupling that gave the correct relic density. We found that in some regions of parameter space, DM would be difficult to observe in direct detection experiments while it would be easier to observe in indirect detection experiments while for other regions of parameter space the situation was reversed. Thus, measurements from both types of experiments complement each other and could help pinpoint the details of the hidden SU(3) model.
\end{abstract}

XXIX International Symposium on Lepton Photon Interactions at High Energies - LeptonPhoton2019 August 5-10, 2019

Toronto, Canada

${ }^{*}$ Speaker. 


\section{Introduction}

There is evidence that dark matter (DM) makes up a significant fraction of the total matter in the universe but virtually nothing is known about it except its relic abundance. While weakly interacting massive particle (WIMP) DM is an attractive paradigm, the simplest models of WIMP DM are either significantly constrained or entirely ruled out [1]. In this contribution, we describe the phenomenological implications of a more complicated model, the multispecies hidden SU(3) model of Arcadi et al [2]. A more detailed report of this analysis is given in [3]. For the parameter space we focus on, the model has 4-7 DM species consisting of scalar and vector DM. We find that the vector particles can more readily be observed in direct detection while the scalar particles are more likely to be observed in indirect detection measurements.

\section{The Hidden Gauged SU(3) Model}

We study the phenomenology of the Hidden Gauged SU(3) model of Arcadi et al. [2] which consists of spin- 1 and spin- 0 states. In their paper, they examined two representative limiting cases that made their numerical analysis more tractable. We extend their studies to consider different points in the parameter space that leads to more complex DM scenarios [3].

The model consists of a gauged SU(3) which is fully broken by two complex scalar triplets, $\Phi_{1}$ and $\Phi_{2}$, so that all the new gauge bosons acquire a mass. These new scalars are not charged under the SM gauge groups and can only interact with the SM through the SM Higgs doublet. To simplify the Lagrangian and insure additional stable states, a $\mathbb{Z}_{2}$ symmetry is also imposed such that $\Phi_{1} \rightarrow-\Phi_{1}$ and $\Phi_{2} \rightarrow \Phi_{2}$, which has the effect of only including even powers of the scalar triplets in the Lagrangian. Following Ref. [2], the Lagrangian is given by:

$$
\mathscr{L}=\mathscr{L}_{\mathrm{SM}}+\mathscr{L}_{\text {portal }}+\mathscr{L}_{\text {hidden }}
$$

where $\mathscr{L}_{\text {portal }}$ and $\mathscr{L}_{\text {hidden }}$ are the new DM pieces given by

$$
\begin{aligned}
-\mathscr{L}_{\text {portal }}= & V_{\text {portal }}=\lambda_{H 11}|H|^{2}\left|\Phi_{1}\right|^{2}+\lambda_{H 22}|H|^{2}\left|\Phi_{2}\right|^{2} \\
-\mathscr{L}_{\text {hidden }}=- & \frac{1}{4} G_{\mu \nu}^{a} G^{\mu v a}+\left|D_{\mu} \Phi_{1}\right|^{2}+\left|D_{\mu} \Phi_{2}\right|^{2}-m_{11}^{2}\left|\Phi_{1}\right|^{2}-m_{22}^{2}\left|\Phi_{2}\right|^{2}-\frac{\lambda_{1}}{2}\left|\Phi_{1}\right|^{4}-\frac{\lambda_{2}}{2}\left|\Phi_{2}\right|^{4} \\
& \quad-\lambda_{3}\left|\Phi_{1}\right|^{2}\left|\Phi_{2}\right|^{2}-\lambda_{4}\left|\Phi_{1}^{\dagger} \Phi_{2}\right|^{2}-\frac{\lambda_{5}}{2}\left[\left(\Phi_{1}^{\dagger} \Phi_{2}\right)^{2}+h . c\right] .
\end{aligned}
$$

Here, $G_{\mu \nu}^{a}=\partial_{\mu} A_{v}^{a}-\partial_{v} A_{\mu}^{a}+\tilde{g} f^{a b c} A_{\mu}^{b} A_{v}^{c}$ is the field strength tensor for the hidden SU(3) where $\tilde{g}$ is the gauge coupling and $f^{a b c}$ are the $\mathrm{SU}(3)$ structure constants.

In the scalar sector, working in the unitary gauge and because of the specific choices we made [3], the scalar mass matrix is a $5 \times 5$ matrix where $\Phi^{T}=\left(h, \varphi_{1}, \varphi_{2}, \varphi_{3}, \varphi_{4}\right)$. Due to our choices, $\varphi_{3}$ and $\varphi_{4}$ cannot mix with any of the other scalars and because we do not have $C P$ violation in the scalar sector, they also cannot mix with each other so both are mass eigenstates which we relabel as $\varphi_{3}=\mathscr{H}$ and $\varphi_{4}=\chi$. The remaining $3 \times 3$ mass matrix can be diagonalized using three mixing angles. For $\theta_{2}$ small, one can define an effective mixing angle $\theta=\theta_{1}+\theta_{3}$ which mixes $h$ and $\phi_{2}$. $\theta$ is constrained to be small by the Higgs boson properties [2, 4]. 
In the vector sector, only $A^{3}$ and $A^{8}$ mix and the vector boson masses are given by:

$m_{A^{1^{\prime}}, A^{2^{\prime}}}=\frac{\tilde{g}}{2} v_{2}, m_{A^{4^{\prime}}, A^{5^{\prime}}}=\frac{\tilde{g}}{2} v_{1}, m_{A^{6^{\prime}}, A^{7^{\prime}}}=\frac{\tilde{g}}{2} \sqrt{v_{1}^{2}+v_{2}^{2}}, m_{A^{3^{\prime}}, A^{8^{\prime}}}^{2}=\frac{\tilde{g}^{2}}{6}\left(v_{1}^{2}+v_{2}^{2} \mp \sqrt{v_{1}^{4}-v_{1}^{2} v_{2}^{2}+v_{2}^{4}}\right)$.

Where $v_{1}$ is the vev for the 3rd component of $\Phi_{1}$ and $v_{2}$ and $v_{3}$ are the vevs for the 2nd and 3rd components of $\Phi_{2}$. We can see that $A_{\mu}^{3^{\prime}}$ is the lightest vector boson while $A_{\mu}^{8^{\prime}}$ is the heaviest, or one of the heaviest. When $v_{1}=v_{2}, A_{\mu}^{8^{\prime}}$ becomes degenerate with $A_{\mu}^{6^{\prime}}$ and $A_{\mu}^{7^{\prime}}$.

The model has 3 global $\mathbb{Z}_{2}$ symmetries. Due to these symmetries and the decay kinematics resulting from the masses, the model will have a minimum of 4 stable DM species and a maximum of 7 .

Before applying the experimental constraints, we apply the usual theoretical constraints on the parameters of the model: partial wave unitarity, the scalar potential being bounded from below, and that we avoid alternative minima. These details are outlined in Ref. [3].

\section{Results and Discussion for the Hidden Gauged SU(3) Model}

The core calculation of our analysis is calculating the relic abundance when many species are present. We adapt the coupled Bolzmann equations given by Dienes, Huang and Thomas [5], which can include an arbitrary number of DM species, to include only $2 \rightarrow 2$ and $1 \rightarrow 2$ interactions. Integrating the coupled Boltzmann equations to obtain the relic density for multiple DM species is computationally intensive and because the parameter space of the Hidden Gauged SU(3) model is large, it is impractical to perform a complete scan of the parameter space. Instead we explore the implications of three representative benchmark points. The parameter values for benchmark point A were chosen to explore the region where one of the DM species could annihilate to SM particles through a Higgs resonance. Benchmark point $\mathrm{B}$ was chosen to explore a region where no resonant effects would occur. Finally, benchmark point $\mathrm{C}$ was chosen to explore the region where $v_{1}$ and $v_{2}$ have similar values and where there are many more stable DM particles.

We vary the mass of one of the DM species and find the gauge coupling $\tilde{g}$ which results in the correct relic density. We vary the mass of $\chi, \mathscr{H}$, and $A^{1^{\prime}}$ for benchmark point $\mathrm{A}, \mathrm{B}$, and $\mathrm{C}$, respectively. Some of the hidden sector particles are stable because symmetry respecting decays are kinematically forbidden. Thus, benchmark points A and B have 4 stable DM species, while benchmark point $\mathrm{C}$ has $7 \mathrm{DM}$ species. The benchmark points we consider are defined in Table 1. We ignore any unstable dark sector species by assuming that they would have decayed out long before freezeout occurred. Note that for benchmark point A, there is a disallowed region around half the Higgs mass because the gauge coupling required to give the correct relic abundance violates the unitarity limit for gauge boson scattering.

For DM direct detection, we compare our DM direct detection rate limits to the XENON1T measurements [6]. Because there are multiple DM species, we need to calculate the expected number of events per species and then sum over all species (see Ref. [7] and [3] for details). The rate is calculated with the energy range, detection efficiency and exposure $(1.0 \mathrm{t} \times \mathrm{yr})$ appropriate for the XENON1T experiment which yields the limit on the rate of $1.58 \times 10^{-4} \mathrm{~kg}^{-1} \mathrm{day}^{-1}$ at $95 \%$ C.L.. The predicted rate is dependent on the DM nucleus cross section at zero momentum transfer which in turn is dependent on the cross section for DM species $i$ and a nucleon. This cross section 


\begin{tabular}{|c|c|c|c|}
\hline Parameters & Scenarios A & Scenarios B & Scenarios C \\
\hline \hline$m_{A^{1^{\prime}}}$ & $300 \mathrm{GeV}$ & $300 \mathrm{GeV}$ & $200-500 \mathrm{GeV}$ \\
\hline$m_{h_{2}}$ & $2500 \mathrm{GeV}$ & $2500 \mathrm{GeV}$ & $600 \mathrm{GeV}$ \\
\hline$m_{h_{3}}$ & $650 \mathrm{GeV}$ & $650 \mathrm{GeV}$ & $225 \mathrm{GeV}$ \\
\hline$m_{\mathscr{H}}$ & $1000 \mathrm{GeV}$ & $400-600 \mathrm{GeV}$ & $250 \mathrm{GeV}$ \\
\hline$m_{\chi}$ & $50-200 \mathrm{GeV}$ & $1000 \mathrm{GeV}$ & $250 \mathrm{GeV}$ \\
\hline$v_{1} / v_{2}$ & 10 & 10 & 1.2 \\
\hline $\mathrm{DM}$ & $\chi, A^{1^{\prime}}, A^{2^{\prime}}, A^{3^{\prime}}$ & $\mathscr{H}, A^{1^{\prime}}, A^{2^{\prime}}, A^{3^{\prime}}$ & $\begin{array}{c}\mathscr{H}, \chi, A^{1^{\prime}}, A^{2^{\prime}} \\
A^{3^{\prime}}, A^{4^{\prime}}, A^{5^{\prime}}\end{array}$ \\
\hline
\end{tabular}

Table 1: Input parameters for the benchmark points. $m_{\chi}, m_{\mathscr{H}}$, and $m_{A^{1^{\prime}}}$ were allowed to vary for benchmark point $\mathrm{A}, \mathrm{B}$, and $\mathrm{C}$, respectively, along with the gauge coupling which was varied to obtain the correct relic density. In all cases, we take the mixing in the scalar sector to only be between $h_{1}$ and $h_{3}$ with a mixing angle of $\sin \theta=0.1$. We also set $m_{h_{1}}$ equal to the SM Higgs mass.

is mediated by two scalars, $h_{1}$ and $h_{3}$, so that the cross section for DM species $i$ and a nucleon is given by:

$$
\sigma_{i n}^{0}=\frac{f_{N}^{2}}{4 \pi}\left(\frac{g_{i i h_{1}} c_{\theta}}{m_{h_{1}}^{2}}+\frac{g_{i i h_{3}} s_{\theta}}{m_{h_{3}}^{2}}\right)^{2} \frac{m_{n}^{2}}{\left(m_{n}+m_{i}\right)^{2} v^{2}},
$$

where $c_{\theta}$ and $s_{\theta}$ are $\cos \theta$ and $\sin \theta$, respectively; $f_{N} \simeq 0.30 \pm 0.03$ is the SM Higgs effective coupling to nucleons; $m_{n}$ is the nucleon mass; $m_{i}$ is the mass of the DM species in question; $v$ is the SM vev; and $g_{i i h_{1}}$ and $g_{i i h_{3}}$ are the couplings between the DM and $h_{1}$ and $h_{3}$, respectively. The predicted rates along with the XENON1T limit are shown in Fig. 1. The scalar DM, $\chi$ and $\mathscr{H}$, interact weakly with nucleons because the scalar- $h_{1}$ coupling is small and $h_{3}$ interacts with nucleons via its SM Higgs component which is suppressed by the small mixing angle. As a consequence, when the DM is mainly scalar it is weakly constrained by direct detection.

For DM indirect detection, we compare our predictions to the DM cross section limits given by the Fermi-LAT collaboration [8] . Only the $W^{+} W^{-}$final states provided useful constraints [3]. We obtained bounds by scaling the predicted cross sections for DM species $i$ by its fractional component of the relic abundance:

$$
\sigma_{\text {scaled }}=\left(\frac{\Omega_{i}}{\Omega_{D M}}\right)^{2} \frac{\left(\sigma_{i i \rightarrow S M+S M}\right)^{2}}{\sigma_{i i}^{t o t}},
$$

where $\sigma_{i i \rightarrow S M+S M}$ is the cross section from two identical DM particles to one of the SM final states considered by the Fermi collaboration and $\sigma_{i i}^{t o t}$ is the sum of the cross sections to all possible final states. The results for each of the benchmark points are shown in Fig. 1. In all cases the scalar DM has a significantly larger scaled cross section to $W^{+} W^{-}$than any vector DM because the vector DM annihilates preferentially to other DM particles. At present, indirect detection does not impose any constraints although in all three cases, there is the potential to rule out large regions of parameter space with moderate improvements to the experimental bounds.

One sees from Fig.1 that for benchmark point B, which is mainly scalar DM, the predicted direct detection cross sections are significantly below the XENON1T limits but close enough to the 
indirect detection limits that they can potentially be probed in the foreseeable future. In contrast, benchmark $\mathrm{C}$ is mainly vector and is almost entirely ruled out by direct detection limits. Benchmark point $\mathrm{A}$ is interesting as in some regions it is mainly scalar while in other regions mainly vector. When the DM is mainly vector it is ruled out by direct detection limits, but is far below the indirect detection limits, while when it is mainly scalar, the opposite happens.

\section{Summary}

We reported on a study [3] of the hidden SU(3) DM model of Arcadi et al [2] which has multiple vector and scalar DM species. We used a coupled Boltzmann equation that can incorporate arbitrary numbers of DM species to calculate the relic abundance [5]. We focused on 3 benchmark points with 4 and 7 DM species. In general, it is important to include all stable particles in the freeze-out calculations as interactions with species with even small particle densities can significantly alter the final results. We studied the sensitivity of direct and indirect detection measurements to the DM and found that vector DM is best constrained by direct detection measurements while scalar DM is best constrained by indirect detection measurements. This is a reminder of the complementarity of the different measurements. It also points out that with complicated DM sectors, it is possible to obtain the correct relic abundance but still not be able to observe it with these measurements.

\section{Acknowledgments}

We thank Keith Dienes and Brooks Thomas for helpful conversations and communications. This work was supported by the Natural Sciences and Engineering Research Council of Canada.

\section{References}

[1] G. Arcadi, M. Dutra, P. Ghosh, M. Lindner, Y. Mambrini, M. Pierre, S. Profumo and F. S. Queiroz, The waning of the WIMP? A review of models, searches, and constraints, Eur. Phys. J. C 78 (2018) $203[\operatorname{arXiv}: 1703.07364$ [hep-ph]].

[2] G. Arcadi, C. Gross, O. Lebedev, Y. Mambrini, S. Pokorski and T. Toma, Multicomponent Dark Matter from Gauge Symmetry, JHEP 1612 (2016) 081 [arXiv:1611.00365 [hep-ph] ].

[3] A. Poulin and S. Godfrey, Multicomponent dark matter from a hidden gauged SU(3), Phys. Rev. D 99 (2019) 076008 [arXiv:1808.04901 [hep-ph]].

[4] A. Falkowski, C. Gross and O. Lebedev, A second Higgs from the Higgs portal, JHEP 1505 (2015) $057[\operatorname{arXiv}: 1502.01361$ [hep-ph]].

[5] K.R. Dienes, F. Huang, and B. Thomas, to appear.

[6] E. Aprile et al., Dark Matter Search Results from a One Tonne $\times$ Year Exposure of XENON1T, Phys. Rev. Lett. 121 (2018) 111302 [arXiv:1805.12562 [astro-ph]].

[7] K. Dienes, J. Kumar and B. Thomas, Direct Detection of Dynamical Dark Matter, Phys. Rev. D 86

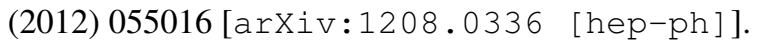

[8] M. Ackermann et al. [Fermi-LAT Collaboration], Searching for Dark Matter Annihilation from Milky Way Dwarf Spheroidal Galaxies with Six Years of Fermi Large Area Telescope Data, Phys. Rev. Lett. 115 (2015) 231301 [arXiv: 1503.02641 [astro-ph.HE]]. 

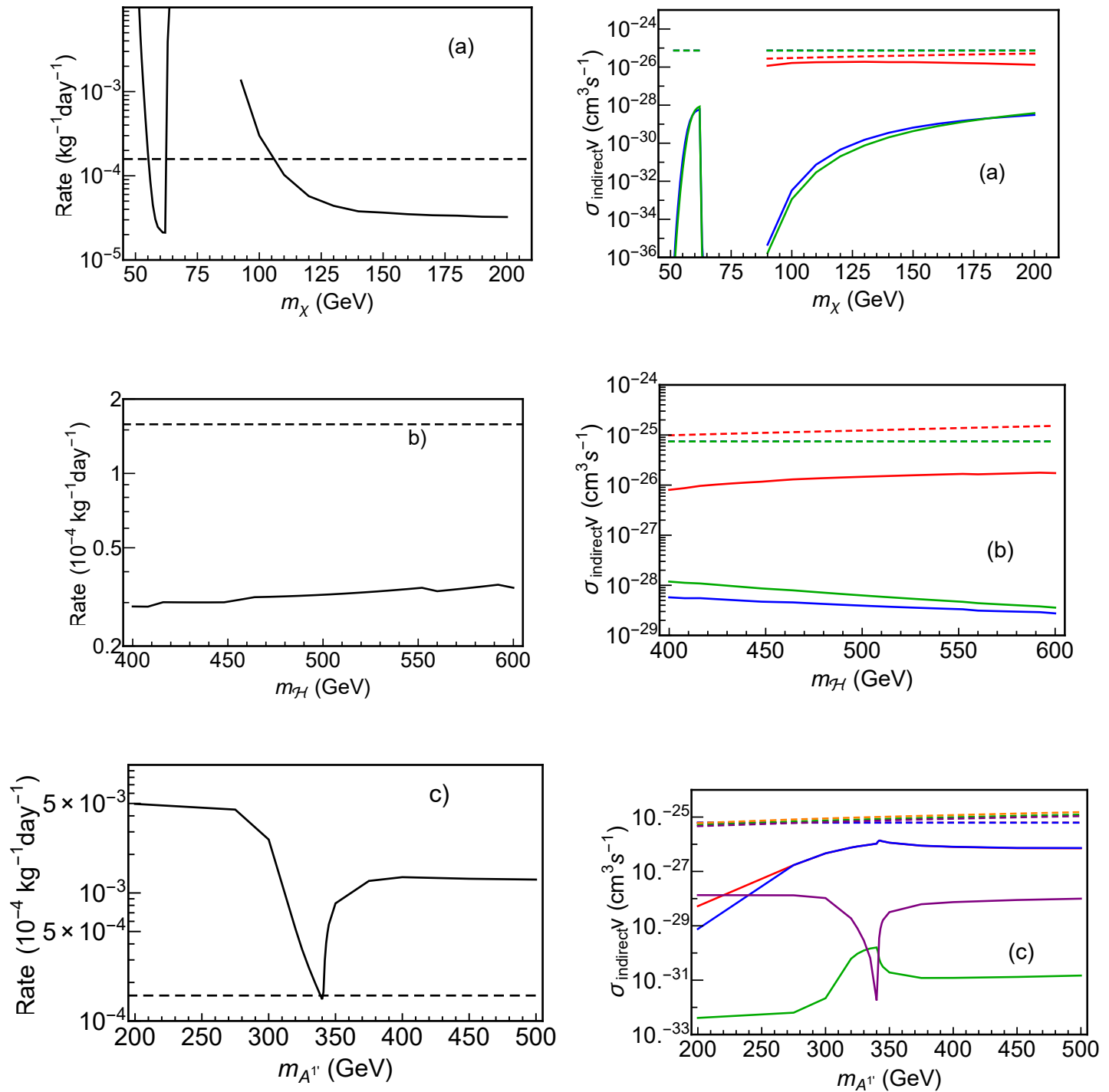

Figure 1: The figures on the left are the direct detection event rates as a function of the DM mass and the figures on the right are the indirect detection constraints for the $W^{+} W^{-}$final state. In both cases the plots (a), (b), and (c) show the results for benchmark point A, B and C, respectively. For direct detection, the allowed points are below the dashed line at $1.58 \times 10^{-4} \mathrm{~kg}^{-1} \mathrm{day}^{-1}$. For the indirect detection plots the line colors represent: red for the $\chi \chi \rightarrow W^{+} W^{-}$, blue for $\mathscr{H} \mathscr{H} \rightarrow W^{+} W^{-}$, green for $A^{1^{\prime}} A^{1^{\prime}} \rightarrow W^{+} W^{-}$or $A^{2^{\prime}} A^{2^{\prime}} \rightarrow W^{+} W^{-}$which have identical results, purple for $A^{3^{\prime}} A^{3^{\prime}} \rightarrow W^{+} W^{-}$, and orange for $A^{4^{\prime}} A^{4^{\prime}} \rightarrow W^{+} W^{-}$ or $A^{5^{\prime}} A^{5^{\prime}} \rightarrow W^{+} W^{-}$which have identical results. The dashed lines are the constraints from Fermi-LAT [8] while the solid lines are the predictions of the model. Note that the purple and green dashed line are almost superimposed because they have very similar masses leading to similar constraints. We also note that the solid orange line does not appear because it is too small. 